\title{
Coexistence of Two Families of Sub-Harmonic Resonances in a Time-Delayed Nonlinear System at Different Forcing Frequencies
}

\author{
J.C. Ji \\ School of Electrical, Mechanical and Mechatronic Systems \\ Faculty of Engineering and IT, University of Technology Sydney \\ PO Box 123, Broadway, NSW 2007, Australia \\ Phone: (+61 2) $95142677 . \quad$ Email: jin.ji@uts.edu.au \\ Jin Zhou \\ Shanghai Institute of Applied Mathematics and Mechanics, Shanghai University \\ Shanghai 200072, PR China
}

\begin{abstract}
Two coexisting families of sub-harmonic resonances can be induced at different forcing frequencies in a time-delayed nonlinear system having quadratic nonlinearities. They occur in the region where two stable bifurcating periodic solutions coexist in the corresponding autonomous system following two-to-one resonant Hopf bifurcations of the trivial equilibrium. The forced response is found to demonstrate small- and largeamplitude quasi-periodic motion under the family of sub-harmonic resonances related to Hopf bifurcation frequencies, and large-amplitude periodic and quasi-periodic motion under the family of sub-harmonic resonances associated with the shifted Hopf bifurcation frequencies. The family of sub-harmonic resonances related to Hopf bifurcation frequencies may cease to exist with the loss of the initially established frequency relationship of sub-harmonic resonances when the magnitude of periodic excitation is beyond a certain value. This will lead to a jump phenomenon from small- to largeamplitude quasi-periodic motion. Bifurcation diagrams, time trajectories and frequency spectra are numerically obtained to characterize the sub-harmonic resonances of the time-delayed nonlinear system around the critical point of the resonant Hopf bifurcations.
\end{abstract}

Keywords: Sub-harmonic resonances, loss of sub-harmonic resonance, time-delayed nonlinear system, two-to-one resonant Hopf bifurcations, quasi-periodic motion.

\section{Introduction}


Time delays can inherently appear in many engineering systems with feedback control including, magnetic bearing systems [1], manufacturing process [2-6], microelectromechanical systems [7], actively controlled mechanical systems [8], vehicle systems [9, 10], and spacecraft [11]. Such systems have been referred to as timedelayed nonlinear systems in order to distinguish them from conventional nonlinear systems without time delay, and concurrently time-delayed nonlinear equations used to represent the corresponding mathematical models [12]. Generally speaking, there are three issues needed to be addressed for time-delayed nonlinear systems, which are 1) determination of the critical values of time delays where the trivial equilibrium will lose its stability, 2) identification of bifurcation behaviours of the trivial equilibrium, and 3) characterization of the forced response resulting from the dynamic interactions between the periodic excitation and stable bifurcating solutions (SBSs) generated from Hopf bifurcations. The first two issues can be classified as local stability and bifurcations and are related to the autonomous time-delayed nonlinear systems (no excitation is presented in the system), while the third issue as dynamic interactions and is for the nonautonomous time-delayed nonlinear systems.

Many researchers have investigated the local stability and bifurcations of the autonomous time-delayed nonlinear systems which can exhibit different behaviour from the conventional nonlinear systems. Specifically, the trivial equilibrium of autonomous timedelayed nonlinear systems may lose its stability via single or double Hopf bifurcations when the characteristic equation has one or two pairs of purely imaginary eigenvalues [13-17]. The postcritical behaviour of the time-delayed nonlinear systems may exhibit stable periodic motion or unstable divergent motion following single Hopf bifurcations. The frequency of stable bifurcating periodic solution is related to the frequency of Hopf bifurcations which is different from the so-called linearized natural frequency of the timedelayed nonlinear systems. An interaction of double Hopf bifurcations may generate nonresonant or resonant Hopf bifurcations at the bifurcation point of co-dimension two, depending on the frequency ratio of two Hopf bifurcations. Resonant Hopf bifurcations can lead to interactions of the SBSs which produce more complicated behaviour.

The periodic excitation presented in non-autonomous time-delayed nonlinear systems can dynamically interact with the SBSs. The resultant dynamic interactions can induce resonant oscillations in the forced response and are not yet fully explored for timedelayed nonlinear systems. Primary and secondary resonances were studied for the time-delayed nonlinear systems in the neighbourhood of single Hopf bifurcations [18-21]. 
Additionally, combination resonances such as additive and difference types were found to exist in the neighbourhood of non-resonant Hopf bifurcations [22]. In other words, only one family of resonances was found for the time-delayed nonlinear systems considered in the existing studies. Resonant Hopf bifurcations of the trivial equilibrium can induce more complex dynamic behaviours than single or non-resonant Hopf bifurcations. For example, they can induce the co-existence of two SBSs in the autonomous time-delayed nonlinear system [23]. The dynamic interactions between the periodic excitation and either of the $S B S s$ can create different families of resonances in the corresponding non-autonomous system at different forcing frequencies (will be briefly discussed in Subsection 2.3).

This paper will study two families of sub-harmonic resonance response in a time-delayed quadratic nonlinear system. The coexistence of sub-harmonic resonances in a timedelayed nonlinear system can be considered as a new dynamic phenomenon that has not yet been reported in the literature. It has no analogy in one degree-of-freedom conventional nonlinear system with only one external excitation, because the conventional system has only one type of sub-harmonic resonances depending on the order of nonlinear terms and the forcing frequency. The cascades of combined bifurcations (resonances) may be only possible for one degree-of-freedom conventional nonlinear system with two external periodic excitations, as discussed for a Duffing's oscillator with two external periodic forces in reference [24]. It should be noted that the combination resonances in classical nonlinear oscillator with two excitations occur when the two forcing frequencies satisfy certain relationships with the system's linearized natural frequency. On the contrary, the combination resonances happen in a timeddelayed nonlinear system of one-degree-of-freedom having only one periodic excitation when the two frequencies of bifurcating periodic solutions satisfy certain relationships with the forcing frequency.

The remainder of this paper is organized as follows. Section 2 briefly discusses two coexisting SBSs and different families of primary and secondary resonances. Two families of sub-harmonic resonance response are numerically studied in Sections 3 and 4. Section 5 presents the concluding remarks.

\section{Coexistence of the SBSs and different families of resonances}

This section provides a brief background on the resonant Hopf bifurcations, the coexistence of the SBSs, the approximate solutions to the family of sub-harmonic 
resonance response related to Hopf bifurcation frequencies, and then introduces the different families of resonances.

\subsection{The time-delayed nonlinear system and resonant Hopf bifurcations}

A mass-damper-spring system with feedback control, as shown in Figure 1, can be regarded as a simple model for describing the oscillations of time-delayed nonlinear mechanical systems. The nonlinear spring has a linear-plus-quadratic characteristic stiffness. The quadratic nonlinearities are commonplace in engineering systems and may come from nonlinear elastic forces, nonlinear stress-strain relationships, and geometrical deformations such as initial curvatures and buckled states of flexible structures. The vertical tire force of a vehicle is also a quadratic nonlinear function of the vertical tire deflection in the first approximation. The time delay is considered as delayed reactions of actuators or purposely introduced in the control loop. Periodic excitations considered may come from the eccentricity of unbalance and inertia effects in mechanical systems.

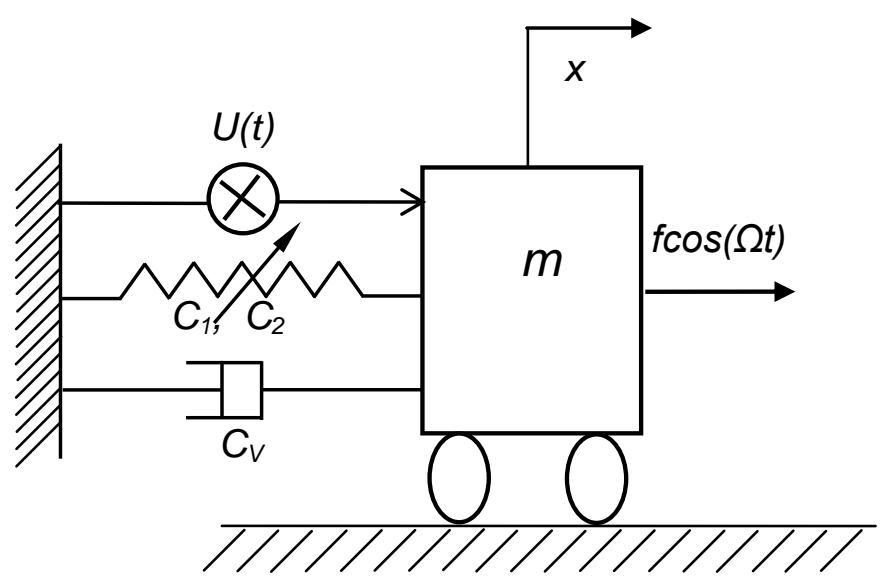

Fig.1. The schematic of a simple time-delayed nonlinear mechanical system.

The dimensionless equation of motion determining the oscillations of the mass can be given by:

$$
\ddot{x}+\mu \dot{x}+\omega^{2} x+\alpha x^{2}=e \cos (\Omega t)+p x(t-\tau)+q \dot{x}(t-\tau)+k_{1} x^{2}(t-\tau),
$$

where $x$ is the displacement, $\mu$ is the damping coefficient, $\omega$ is the so-called linearized natural frequency, $\alpha$ is the coefficient of the quadratic term, $e$ and $\Omega$ denote the amplitude and frequency of the periodic excitation, and an over-dot represents the differentiation with respect to time $t$. The parameters, $\tau, p, q$, and $k_{1}$ stand for the time 
delay, the linear and quadratic feedback gains, respectively. The procedure for deriving equation (1) can be found in reference [25].

The trivial equilibrium of the corresponding autonomous time-delayed nonlinear system (which can be obtained by letting $e=0.0$ in equation (1)) may lose its stability via single or double Hopf bifurcations, depending on the number of purely imaginary eigenvalues. When two pairs of purely imaginary eigenvalues exist, the frequencies of double Hopf bifurcations can be written as:

$$
\delta_{1,2}^{2}=\frac{1}{2}\left(q^{2}-\mu^{2}+2 \omega^{2} \pm \sqrt{4\left(p^{2}-\omega^{4}\right)+\left(q^{2}-\mu^{2}+2 \omega^{2}\right)^{2}}\right) .
$$

The corresponding critical time delays can be obtained as

$$
\tau_{1 \mathrm{c}, \mathrm{n}}=\frac{s_{1}}{\delta_{1}}+\frac{2 n \pi}{\delta_{1}}, \tau_{2 \mathrm{c}, \mathrm{n}}=\frac{s_{2}}{\delta_{2}}+\frac{2 n \pi}{\delta_{2}}, \quad n=0,1,2 \ldots
$$

where $0 \leq s_{1,2}<2 \pi, \sin s_{1,2}=\frac{\left(-\mu p+q \omega^{2}-q \delta_{1,2}^{2}\right) \delta_{1,2}}{p^{2}+q^{2} \delta_{1,2}^{2}}, \cos s_{1,2}=\frac{p \omega^{2}-p \delta_{1,2}^{2}+\mu q \delta_{1,2}^{2}}{p^{2}+q^{2} \delta_{1,2}^{2}}$.

Depending on the coefficients of linear terms and the critical time delay, the trivial equilibrium of the corresponding autonomous system may lose its stability via two-to-one resonant Hopf bifurcations with $\delta_{1}: \delta_{2} \approx 2: 1$, whose point cannot be expressed analytically but located numerically once the system parameters are given [26].

The parameters used in this paper are given by: $\mu=0.1, \omega=2.4, \alpha=0.6, p_{0}=-2.8$, $q_{0}=-1.025, k_{1}=-0.3, \beta_{1}=-0.03, \beta_{2}=0.021$. Here the linear feedback gains are expressed as $p=p_{0}+\beta_{1}, q=q_{0}+\beta_{2}$, to account for the small local area of the point of double Hopf bifurcations. The parameters $\beta_{1}$ and $\beta_{2}$ denote the perturbations of the critical values of linear feedback gains $p_{0}$ and $q_{0}$ at the resonant Hopf bifurcations. For the given parameters, the frequencies of double Hopf bifurcations and the corresponding critical time delay, calculated from Equations (2) and (3), are found to be $\delta_{1}=3.16252$, $\delta_{2}=1.58634, \tau=2.2728$, respectively. The frequency ratio is given by $\delta_{1}: \delta_{2}=1.9936 \approx 2.0$, thus the double Hopf bifurcations can be regarded as two-to-one resonances with $\delta_{1} \approx 2 \delta_{2}$.

\subsection{Two coexisting SBSs}


Following the trivial equilibrium losing its stability via the resonant Hopf bifurcations, the postcritical behaviour of the corresponding autonomous time-delayed nonlinear system may exhibit the coexistence of two SBSs depending on the initial conditions. Figure 2 shows two coexisting $S B S s$ starting from different initial conditions. Numerical solutions are obtained using dde23 in MATLAB under the initial conditions $h(t)=\left(s_{1}, s_{2}\right)$ for $t \leq \tau$ with $s_{1}, s_{2}$ being constants. These two coexisting SBSs are termed as the small- and large-amplitude $S B S s$ according to the maximum amplitude of vibration. As can be seen from the figure, the small-amplitude SBS has two frequency components, 1.582 and $3.161 \mathrm{rad} / \mathrm{s}$, as given in the spectrum diagram. By considering both the rounded calculation errors and the data sampled frequency, these two frequencies can be regarded as the theoretical Hopf bifurcation frequencies (HBFs), which can be obtained from Equation (2) as $\delta_{1}=3.16252, \delta_{2}=1.58634$. On the contrary, the large-amplitude $S B S$ contains four frequency components with two dominant peaks as shown in Figure 2(b). The two peaks are 1.51 and $3.017 \mathrm{rad} / \mathrm{s}$, which are different from the HBFs and termed as the shifted Hopf bifurcation frequencies ( or the SHBFs for brevity). The two small peaks are the sum of the SHBFS and two times the higher SHBF, respectively. Two SHBFS have approximately a ratio of 1.998 , which can also be considered as two-to-one resonances. Unlike the HBFs, the SHBFs cannot be obtained analytically but only numerically by performing FFT analysis. The two SBSs have their own basins of attraction [23].
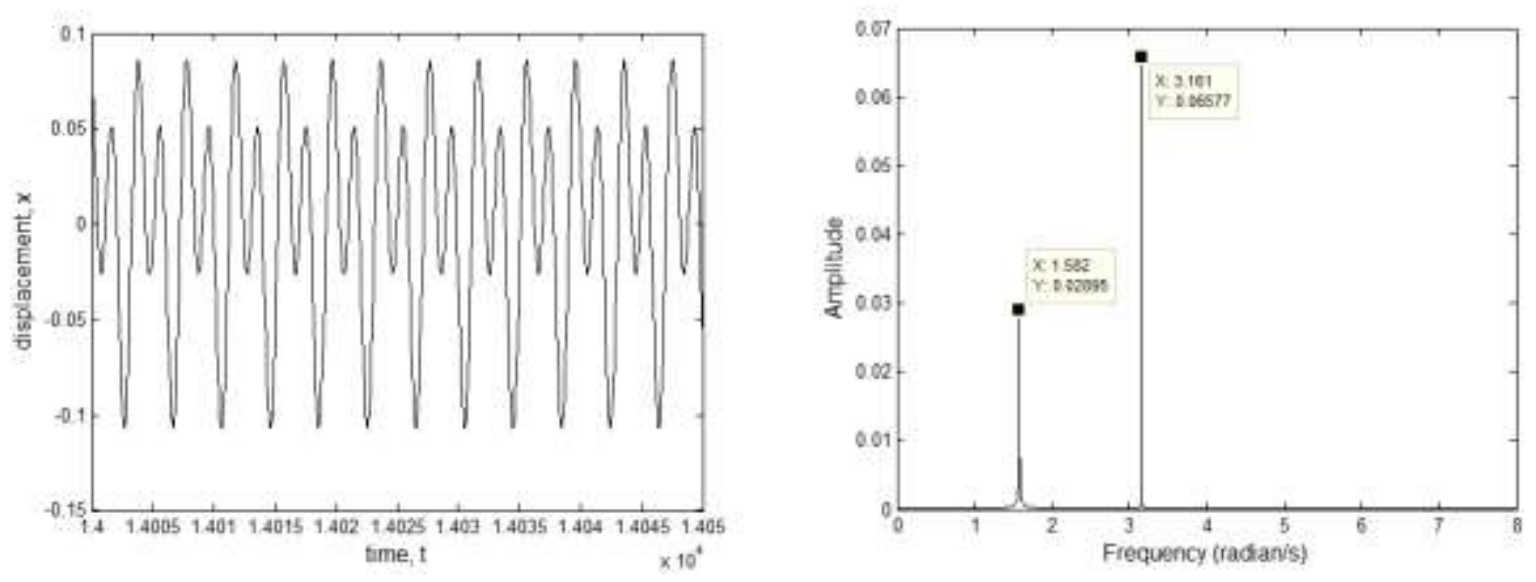

(a) 

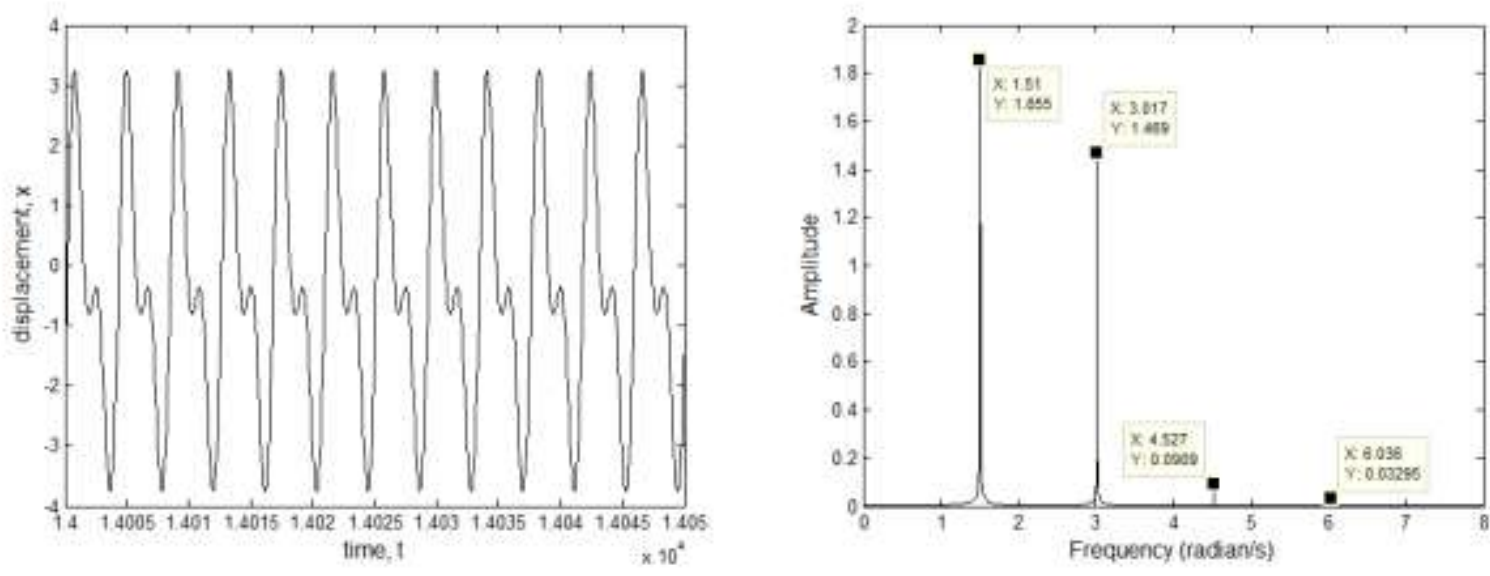

(b)

Fig. 2. The time trajectories and frequency spectra of two SBSs of the corresponding autonomous time-delayed system, (a) the small-amplitude SBS from $h(t)=(0.18,-0.10)$, (b) the large-amplitude SBS from $h(t)=(0.38,-0.10)$.

\subsection{Different families of primary and secondary resonances}

As shown in Sub-section 2.2, the corresponding autonomous time-delayed nonlinear system admits two coexisting SBSs having different frequencies. Dynamic interactions between the periodic excitation and either of SBSs can produce resonances in the forced response of the time-delayed nonlinear system. A total of five types of primary and secondary resonances may occur for each of SBSs. In particular, for the small-amplitude $S B S$ having $H B F s$, the primary resonances can be induced when the forcing frequency is tuned to either of HBFs. In addition, three types of secondary resonances can be generated in the forced response, including sub-harmonic, super-harmonic and combination resonances. The sub-harmonic and super-harmonic resonances associated with the $H B F s$ are expected to happen when the forcing frequency is adjusted at two times the higher $H B F$ and half the lower $H B F$, respectively. The combination resonance related to the HBFs occurs in the form of additive resonances when the forcing frequency is adjusted to be the sum of the HBFs. Similarly, for the large-amplitude SBS having the shifted HBFs, another family of five types of primary and secondary resonances will happen when the forcing frequency is tuned to certain relationships with the SHBFs of the large-amplitude SBS. For the sake of better explanation, a flow chart shown in Figure 3 is used to indicate the different families of primary and secondary resonances. 


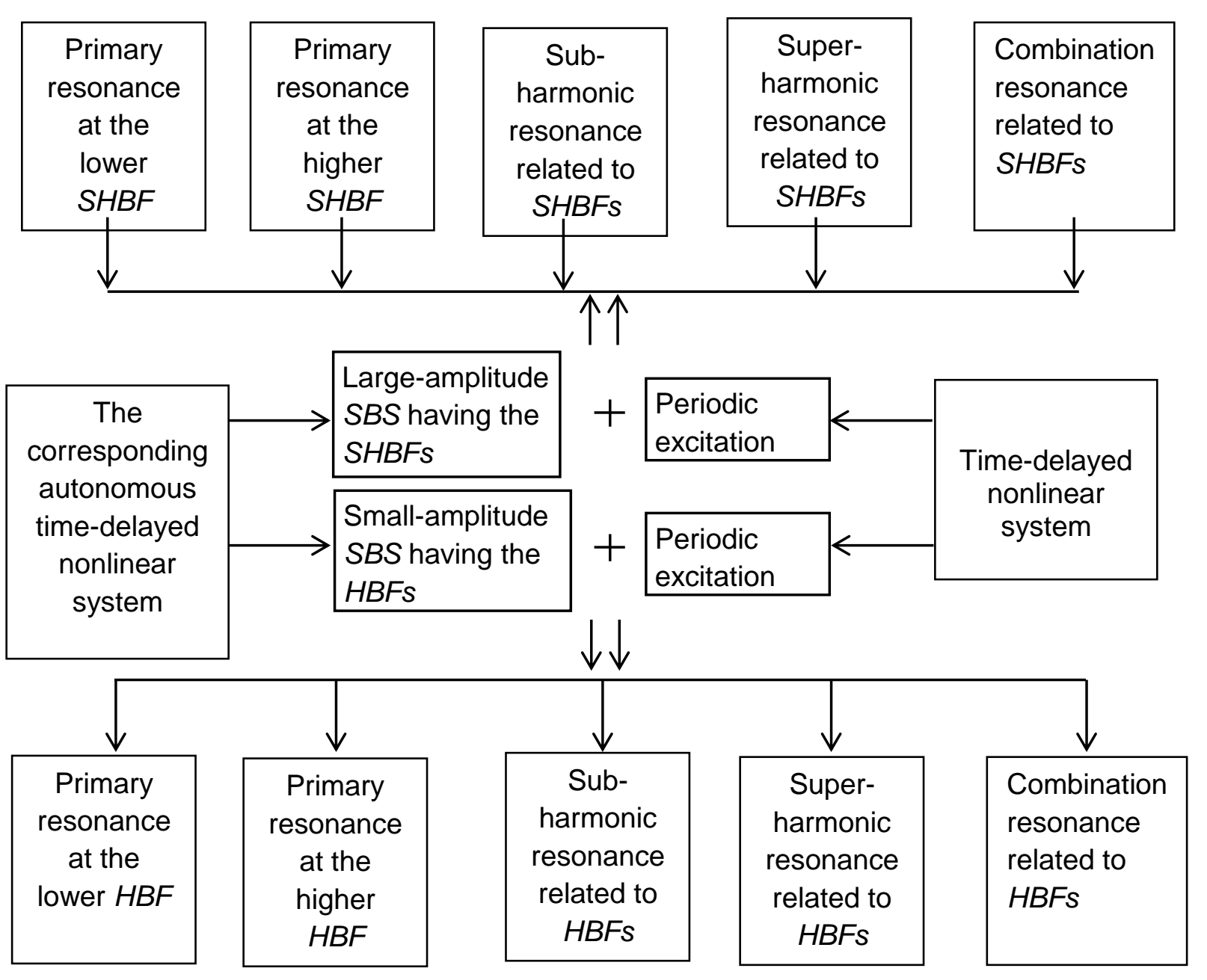

Fig. 3. Block diagram of different resonance families. The terms HBFs and SHBFs represent the Hopf bifurcation frequencies and the shifted Hopf bifurcation frequencies.

According to the reduction theory of functional differential equations [27], the timedelayed nonlinear equation in the neighbourhood of the resonant Hopf bifurcations can be reduced to a system of four first-order ordinary differential equations on the manifold. This indicates that the set of reduced ordinary differential equations is available only for the HBFs but not for the SHBFs. Accordingly, only the approximate solutions to the five types of resonance response related to the HBFs can be developed analytically using a perturbation method, while the approximate solutions to the resonant response associated with the SHBFs cannot be obtained analytically but numerically. The approximate solutions to the secondary resonance response related to the HBFs were analytically constructed and the frequency-response curves were given in reference [25]. Two families of super-harmonic resonances were studied in reference [23] and it was found that one family of super-harmonic resonances may suddenly disappear and the 
other family of super-harmonic resonances can be established by adjusting the forcing frequency accordingly.

\subsection{The analytical approximate solutions}

This subsection briefly recalls the approximate solutions to the sub-harmonic resonance response at two times the higher $H B F$, and then discusses the frequencies involved in the approximate solutions. More details can be found in reference [25].

The set of ordinary differential equations associated with the HBFs is obtained by conducting the reduction of the functional differential equations [27] as:

$$
\begin{aligned}
& \dot{z}_{1}=l_{11} z_{1}+\left(\delta_{1}+l_{12}\right) z_{2}+l_{13} z_{3}+l_{14} z_{4}+f_{10}(z)+e_{1} \cos (\Omega t), \\
& \dot{z}_{2}=\left(-\delta_{1}+l_{21}\right) z_{1}+l_{22} z_{2}+l_{23} z_{3}+l_{24} z_{4}+f_{20}(z)+e_{2} \cos (\Omega t), \\
& \dot{z}_{3}=l_{31} z_{1}+l_{32} z_{2}+l_{33} z_{3}+\left(\delta_{2}+l_{14}\right) z_{4}+f_{30}(z)+e_{3} \cos (\Omega t), \\
& \dot{z}_{4}=l_{41} z_{1}+l_{42} z_{2}+\left(-\delta_{2}+l_{43}\right) z_{3}+l_{44} z_{4}+f_{40}(z)+e_{4} \cos (\Omega t),
\end{aligned}
$$

where $z=\left\{z_{1}, z_{2}, z_{3}, z_{4}\right\}$ represents the local coordinates on the centre manifold associated with the resonant Hopf bifurcations. The coefficients $l_{i j}$ are linear combinations of two small perturbation parameters $\beta_{1}$ and $\beta_{2}$. The nonlinear functions $f_{i 0}(z)$ contain all 10 possible quadratic terms, $z_{i} z_{j}$ with $i, j=1,2,3,4$. The coefficients $l_{i j}$, $e_{i}$, and those in $f_{i 0}(z)$ are not reproduced here.

Equation (4) can be considered as a conventional two-degree-of-freedom weakly quadratic nonlinear system under two-to-one internal resonances. The three frequencies under two-to-one internal resonances and sub-harmonic resonances are assumed to satisfy the relationships:

$$
\delta_{1}=2 \delta_{2}+\varepsilon \sigma_{1}, \Omega=2 \delta_{1}+\varepsilon \sigma_{3}
$$

where $\sigma_{1}$ and $\sigma_{3}$ are the internal and external detuning parameters, respectively.

The first-order approximate solutions which are obtained by using the method of multiple scales [28], can be written as:

$$
\begin{aligned}
& z_{11}=r_{1} \cos \left(\delta_{1} T_{0}+\phi_{1}\right)+A_{1} \cos \left(\Omega T_{0}\right)+A_{2} \sin \left(\Omega T_{0}\right), \\
& z_{21}=-r_{1} \sin \left(\delta_{1} T_{0}+\phi_{1}\right)+A_{3} \cos \left(\Omega T_{0}\right)+A_{4} \sin \left(\Omega T_{0}\right),
\end{aligned}
$$




$$
\begin{aligned}
& z_{31}=r_{2} \cos \left(\delta_{2} T_{0}+\phi_{2}\right)+B_{1} \cos \left(\Omega T_{0}\right)+B_{2} \sin \left(\Omega T_{0}\right), \\
& z_{41}=-r_{2} \sin \left(\delta_{2} T_{0}+\phi_{2}\right)+B_{3} \cos \left(\Omega T_{0}\right)+B_{4} \sin \left(\Omega T_{0}\right),
\end{aligned}
$$

where $r_{1}, r_{2}, \phi_{1}$ and $\phi_{2}$ denote the amplitudes and phases of the free-oscillation terms. The coefficients $A_{i}$ and $B_{i}$ are not reproduced here for brevity (more details can be found in reference [25]).

It should be mentioned that Equation (6) is the approximate solution to the nonlinear equation (4) on the centre manifold. The corresponding approximate solution to the collective (observed) behaviour of the original time-delayed nonlinear system given by equation (1), as a certain combination of solution (6), can be obtained as:

$$
x=r_{1} \cos \left(\frac{1}{2} \Omega t+\frac{1}{2} \varphi_{3}\right)+r_{2} \cos \left(\frac{1}{4} \Omega t-\frac{1}{2} \gamma+\frac{1}{4} \varphi_{3}\right)+\left(A_{1}+B_{1}\right) \cos (\Omega t)+\left(A_{2}+B_{2}\right) \sin (\Omega t),(7)
$$

where the first two terms are the free-oscillation terms and the last two terms are the forced vibration terms. $\varphi_{3}$ and $\gamma$ represent the phases of the free-oscillations terms. Here, the frequencies of two free-oscillation terms are given in terms of the forcing frequency, under the condition that they have been adjusted to a half and a quarter of the forcing frequency by the quadratic nonlinearities involved. Moreover, their ratio becomes exactly two-to-one. On the other hand, if the frequencies cannot be adjusted to the exact relationships of internal and sub-harmonic resonances, then the approximate solutions should be expressed in terms of the HBFs as:

$$
x=r_{1} \cos \left(\delta_{1} t+\phi_{1}\right)+r_{2} \cos \left(\delta_{2} t+\phi_{2}\right)+\left(A_{1}+B_{1}\right) \cos (\Omega t)+\left(A_{2}+B_{2}\right) \sin (\Omega t) .
$$

Given the nature of the nearly commensurable relationship of the two HBFs, the collective response of the time-delayed nonlinear system is quasi-periodic motion, though there may be only three frequencies involved in the motion. This feature is different from the conventional nonlinear systems in that the frequencies of the free-oscillation terms are usually given by the exact relationship of internal and secondary resonances [28].

The three frequency components in the approximate solutions (7) and (8) hold both relationships of internal and sub-harmonic resonances as given by Equation (5). If their relationships are broken in the forced response, then the sub-harmonic resonances will no longer exist. Consequently, the approximate solutions given by equations (7) and (8) will be invalid to represent the sub-harmonic resonance response.

\section{The family of sub-harmonic resonances associated with the HBFs}


While the approximate solutions can be analytically obtained by using a perturbation method for the sub-harmonic response associated with the HBFs, bifurcation diagrams and quasi-periodic motions would be better obtained using numerical methods. In constructing bifurcation diagrams, the steady-state response is obtained by dumping the transient response of the first 16000 periods of the periodic excitation. Then a total of 120 points is taken at each step. The initial condition for numerical integration is $h(t)=(0.18,-0.10)$, from which the corresponding autonomous time-delayed system admits small-amplitude SBS having the HBFs, as discussed in Sub-section 2.2.

\subsection{The forced response}

The forcing frequencies are set to be in the vicinity of two times the higher $H B F$, i.e., 6.322 , under this family of sub-harmonic resonances. Except for the forcing frequency and magnitude, the other system parameters remain unchanged in numerical integration. Figure 4 shows the bifurcation diagrams of the forced response where the excitation magnitude is in the region of $[0.05,3.0]$ with a step of increase by 0.1 . The four forcing frequencies are $\Omega=6.262,6.302,6.342,6.362$, respectively. The bifurcation diagrams can be categorised into two groups according to the outlined shapes, with Figures $4(a, d)$ being classified as Group I and Figures 4(b,c) as Group II.

These two groups of bifurcation diagrams indicate two different patterns of the observed behavior of the time-delayed nonlinear system. By categorizing the maximum vibration amplitude, the forced response exhibits small-amplitude quasi-periodic motion (or simply referred to as small QP motion) for Group I, while small- and large-amplitude quasiperiodic motion (or simply termed as small and large QP motion) for Group II. Unlike Group I, the bifurcation diagrams of Group II are divided into two regions, namely, $0.05 \leq e<0.95$, and $0.95 \leq e \leq 3.5$ in Figure 4 (b), $0.05 \leq e<1.75$, and $1.75 \leq e \leq 3.5$ in Figure 4(c), where small and large QP motion is present, respectively. The small QP motion jumps to large-amplitude non-resonant motion at $e=0.95$ in Figure 4 (b) and at $e=1.75$ in Figure 4(c), respectively. Figures 4(b,c) also imply that jumps occur at different values of the excitation magnitudes for different forcing frequencies. 


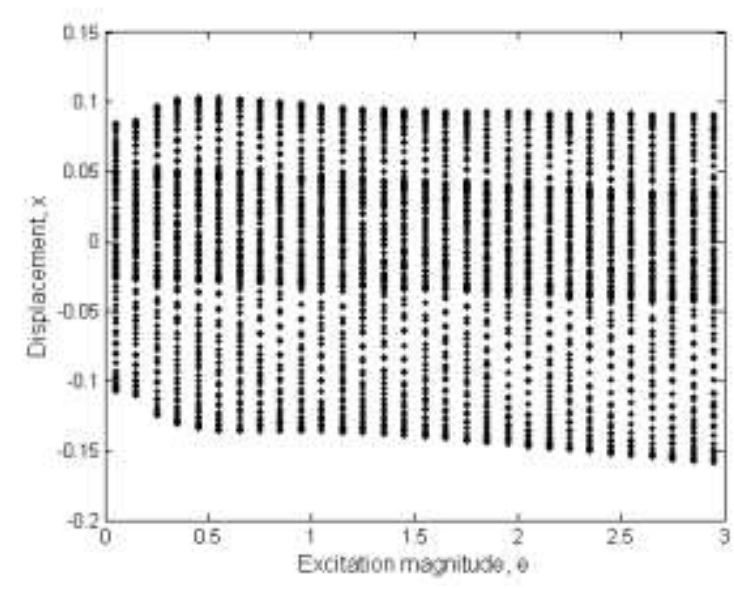

(a) $\Omega=6.262$

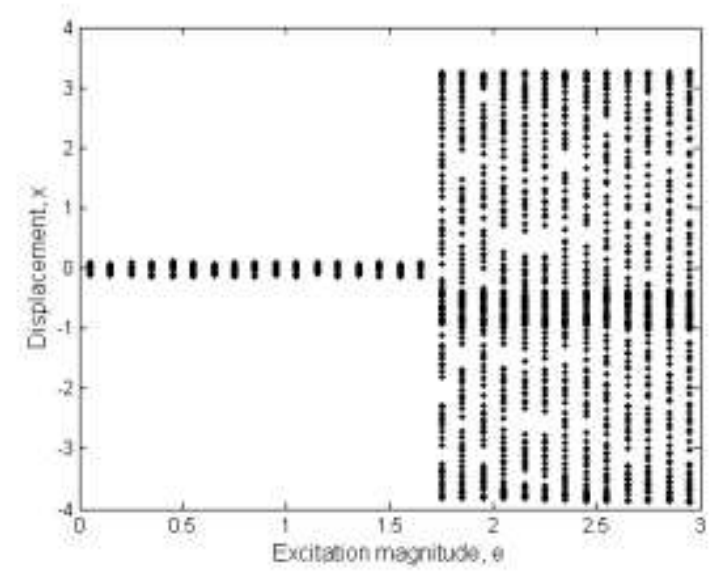

(c) $\Omega=6.342$

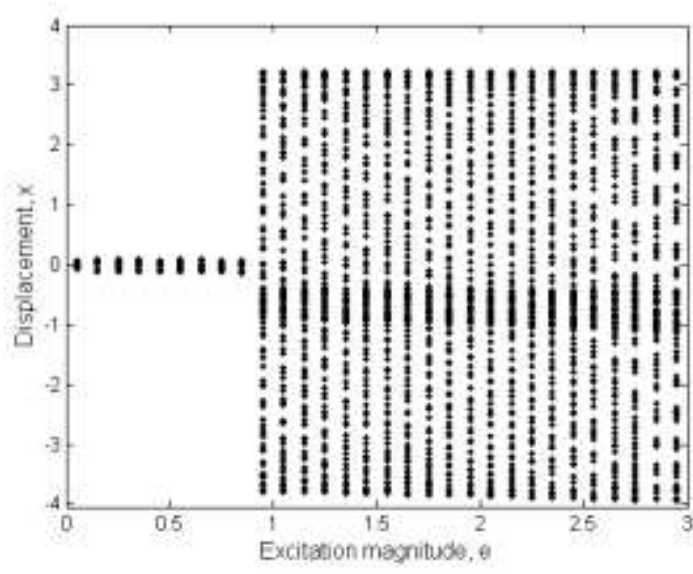

(b) $\Omega=6.302$

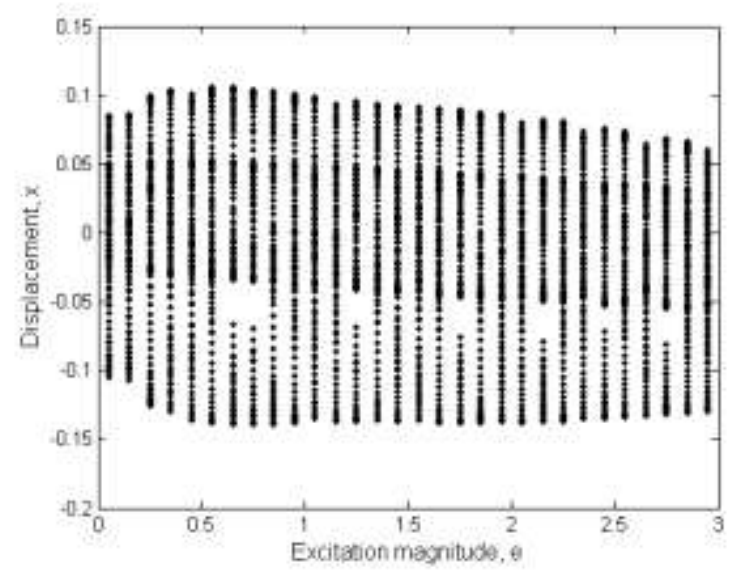

(d) $\Omega=6.362$

Fig. 4. Bifurcation diagrams of the forced response under the sub-harmonic resonance related to the HBFs for different frequencies; (a) $\Omega=6.262$, (b) $\Omega=6.302$, (c) $\Omega=6.342$, (d) $\Omega=6.362$.

The Group I bifurcation diagrams show that the displacements are of small amplitude and change smoothly. Figure 5 gives the small QP motion at $e=2.0$, according to the bifurcation diagram Figure 4(a). The outlined shape of time history shows beat phenomenon, as displayed in Figure 5(a). A close observation indicates three peaks in the time history and the changes in amplitudes of three peaks with time, as shown in Figure 5(b). There are three main frequency components involved in the motion as displayed in Figure 5(c), which are the lower $H B F$, the higher $H B F$, and the forcing frequency, respectively. The phase portrait is a dense cluster of orbits and the corresponding Poincare map shows a closed curve in Figure 5(d), further confirming that the motion is quasi-periodic. The frequencies of the free-oscillation terms are not adjusted to a quarter and half the forcing frequency. A possible explanation would be that the 
small-amplitude SBS and the particular solution generated from the periodic excitation compete each other but the particular solution is not strong enough to adjust the frequency components in the small-amplitude SBS. As the forcing amplitude is increased, the dynamic interaction between the small-amplitude SBS and the particular solution becomes stronger, which is evidenced by the changes in the magnitudes of the HBF components in the response. The magnitude of the forcing frequency component in the forced response increases linearly with a gradual increase of the forcing magnitude. This validates the theoretical predictions of the amplitudes of the particular solution terms given in Equation (8) being linearly proportional to the forcing amplitude.

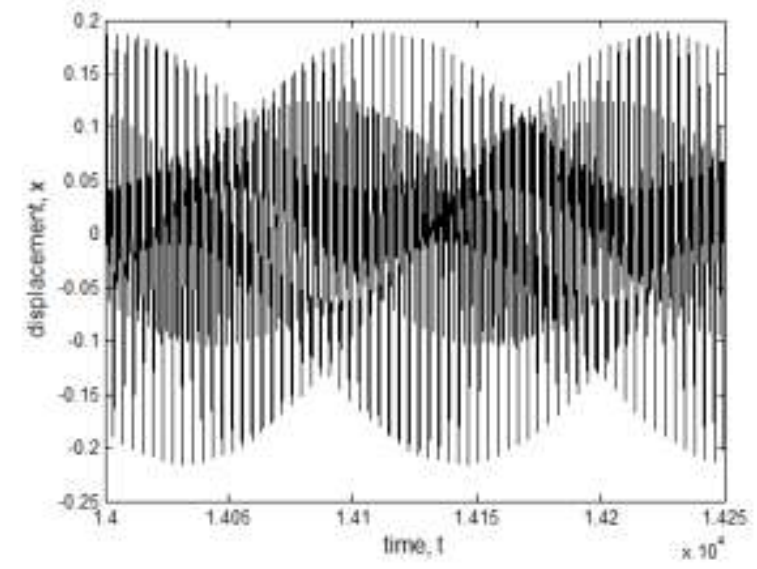

(a)

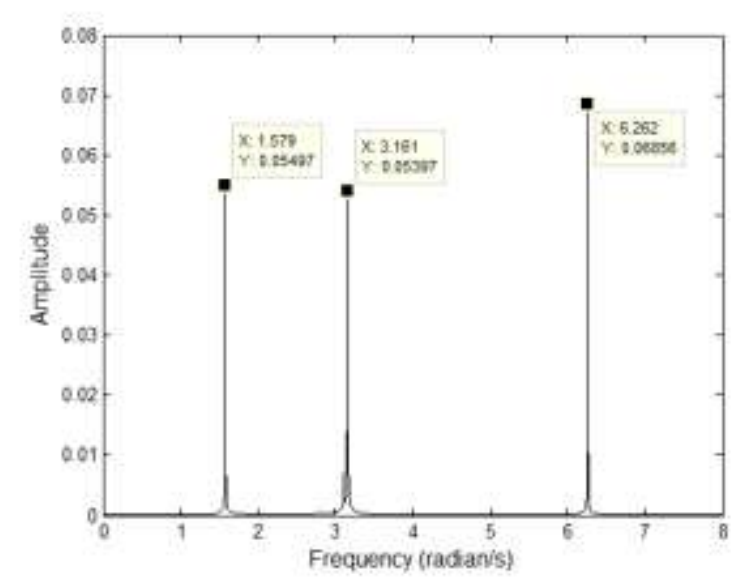

(c)

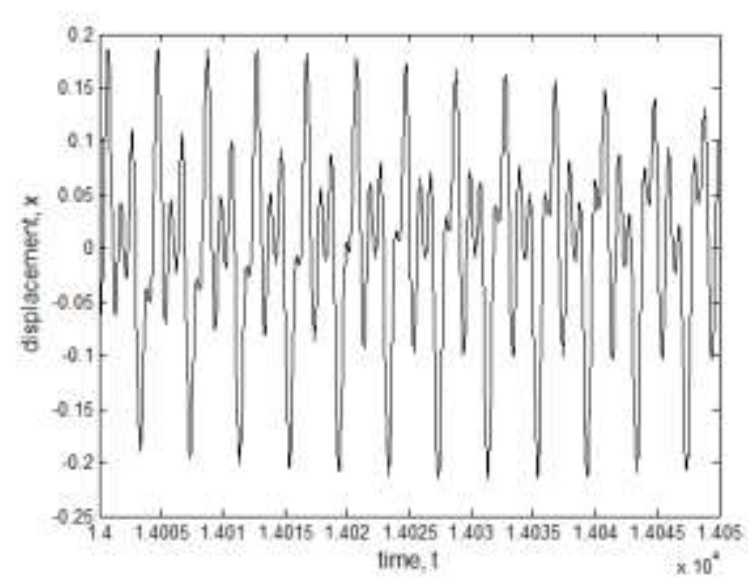

(b)

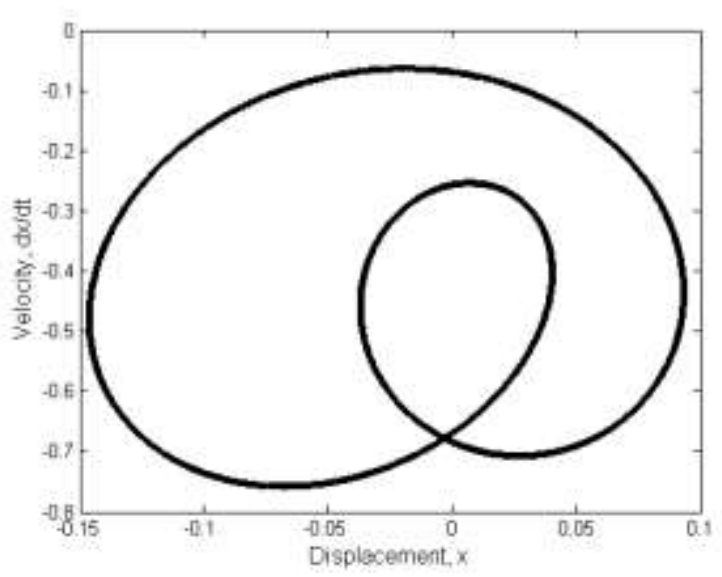

(d)

Fig.5. The sub-harmonic resonance response at $\Omega=6.262$ and $e=2.0$; (a) time history, (b) enlargement of time history, (c) frequency spectrum, (d) Poincare map.

The Group // bifurcation diagrams give a different scenario with two types of motion. The small QP motion disappears and instead large QP motion comes up after the forcing 
magnitude passes a certain value. Simultaneously, the frequency components corresponding to the HBFs vanish and new frequency components related to the SHBFS arise in the forced response. As will be discussed in the next sub-section, this will result in the loss of the sub-harmonic resonance.

\subsection{The loss of the sub-harmonic resonances at two times the higher HBF}

For certain forcing frequencies (for example, as shown in Group // bifurcation diagrams), the forced response may exhibit small or large QP motion depending on the level of the excitation magnitude. The characteristics of two types of quasi-periodic motion are further studied by examining time trajectories and frequency spectra before and after the critical value of the forcing magnitude. According to Figure 4(c) for the forcing frequency $\Omega=6.342$, the forced response exhibits small QP motion at $e=1.65$, as shown in Figure 6(a), with its maximum amplitude being 0.1458 . This amplitude can be considered to be small when compared with that (approximately 3.376 ) of the subsequent quasi-periodic motion given in Figure 6(b). The small QP motion contains three frequency components, which are two HBFs and the forcing frequency, indicating the occurrence of the subharmonic resonance associated with the HBFs. At $e=1.85$, the forced response shows the large QP motion containing seven frequency components, as shown in the frequency spectrum of Figure 6(b). The three dominant peaks are 1.51, 3.017, and $6.343 \mathrm{rad} / \mathrm{s}$, which correspond to the SHBFs and the forcing frequency. The other peaks (lower peaks) are the combinations of these components. The frequency components associated with the HBFs have disappeared. Thus the frequency relationship of the subharmonic resonance at two times the higher HBF no longer exists in the large QP motion. This may suggest that the large-amplitude $S B S$ be excited in the forced response at an expense of the disappearance of the small-amplitude $S B S$. As a result, the initially established sub-harmonic resonance at two times the higher HBF is lost, leading to nonresonant response in the observed behaviour.

The collective behaviour of the time-delayed nonlinear system remains non-resonant response of large-amplitude motion as the forcing magnitude increases further. Figure 6(c) shows the forced response for the excitation amplitude $e=3.5$. The non-resonant response has seven frequency components and the three dominant frequency peaks are two SHBFs and the forcing frequency, that is, $1.507,3.014$, and $6.343 \mathrm{rad} / \mathrm{s}$, respectively. The two SHBFs seem to be under exact two-to-one relationship. The other lower frequency peaks are the combinations of these three dominant frequencies. It is 
interesting to notice that the maximum amplitude of the large QP motion does not increase significantly when compared with that for $e=1.85$, but the magnitudes of the frequency components change, indicating the vibrational energy exchanges between the two frequency modes.
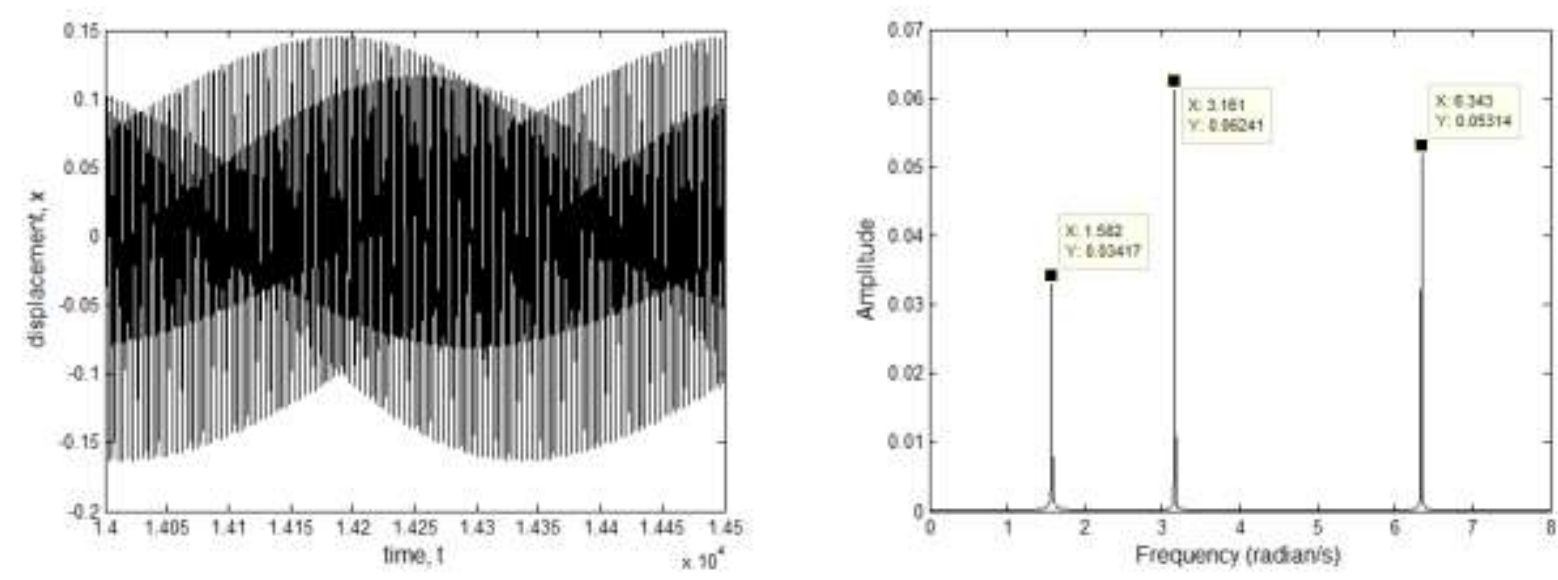

(a) $e=1.65$
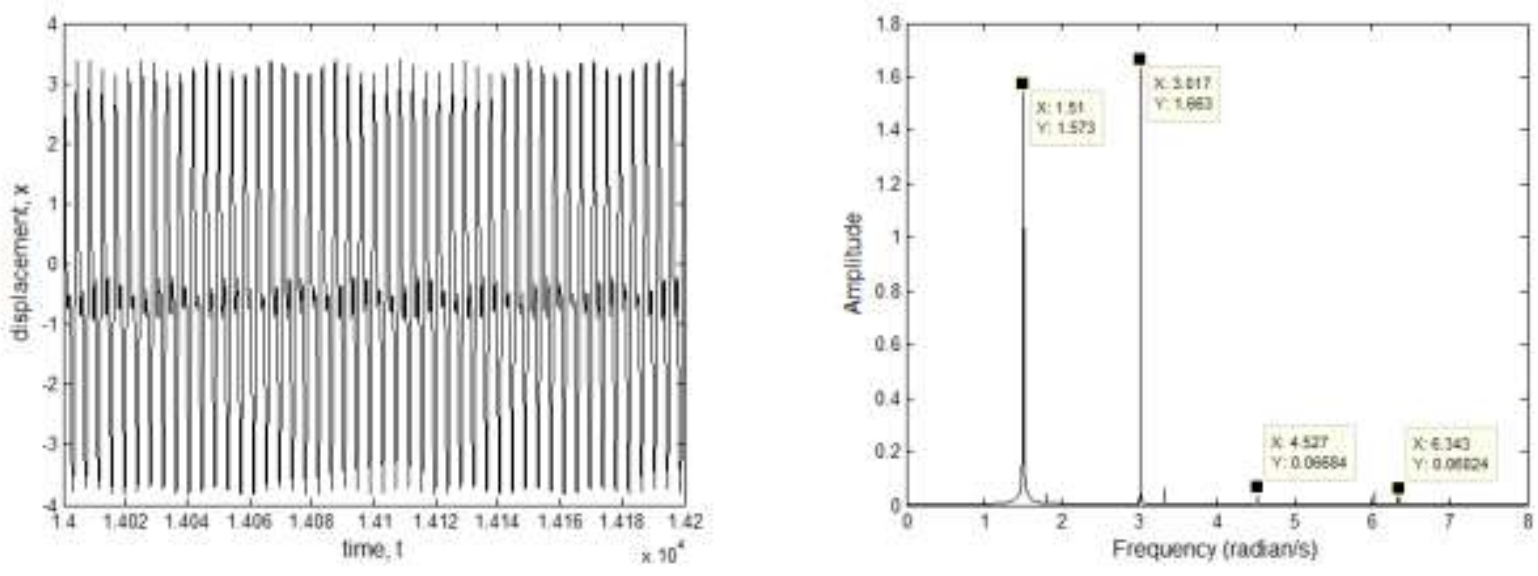

(b) $e=1.85$
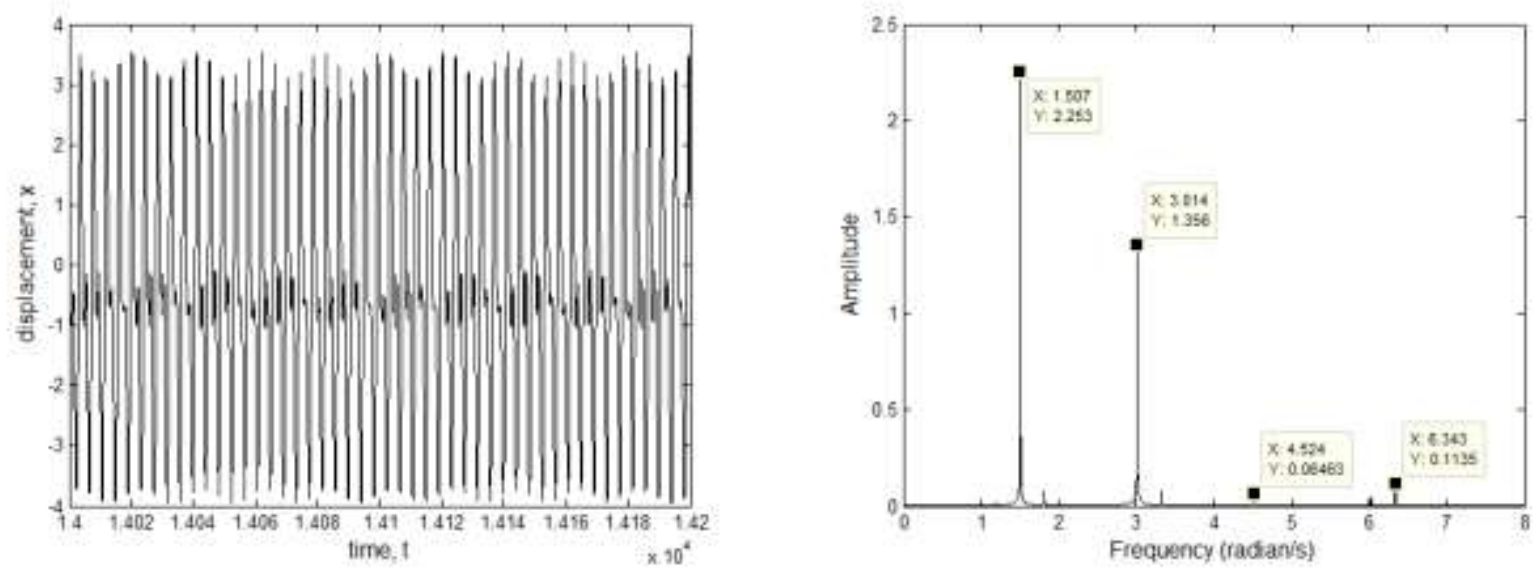

(c) $e=3.5$ 
Fig.6. Time trajectories and frequency spectra of the response at the forcing frequency $\Omega=6.342$ for different excitation magnitudes, (a) $e=1.65$, (b) $e=1.85$, (c) $e=3.5$.

As shown in Figure 4(b), after the excitation magnitude $e>0.95$, the time-delayed nonlinear system exhibits large-amplitude non-resonant response and consists of the SHBFs. If the excitation frequency is then accordingly tunned in line with the newly appeared frequency components (i.e., the $S H B F s$ ), it is found that the other family of the sub-harmonic resonances (i.e., the sub-harmonic resonance associated with the SHBFs) cannot established halfway through. This can be seen from Figures $7(a, b)$, showing the bifurcation diagrams of the forced response with an increase of the forcing amplitude starting from $e=0.95$. In numerically constructing the bifurcation diagrams, only the forcing frequency is changed by choosing a certain value around two times the higher $S H B F$, while the simulation procedure and the other parameters remain unchanged as those for constructing Figure 4(b). The forcing frequency is tuned to be approximately the two times the higher SHBF halfway through.

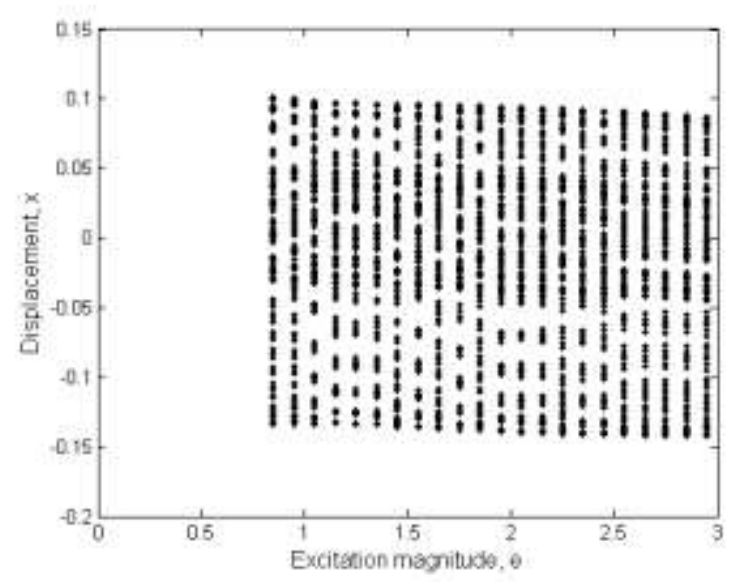

(a) bifurcation diagram at $\Omega=6.034$

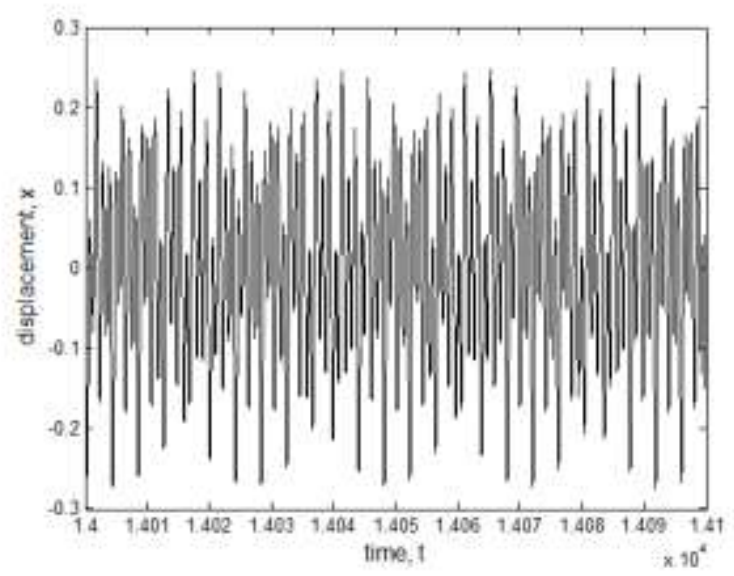

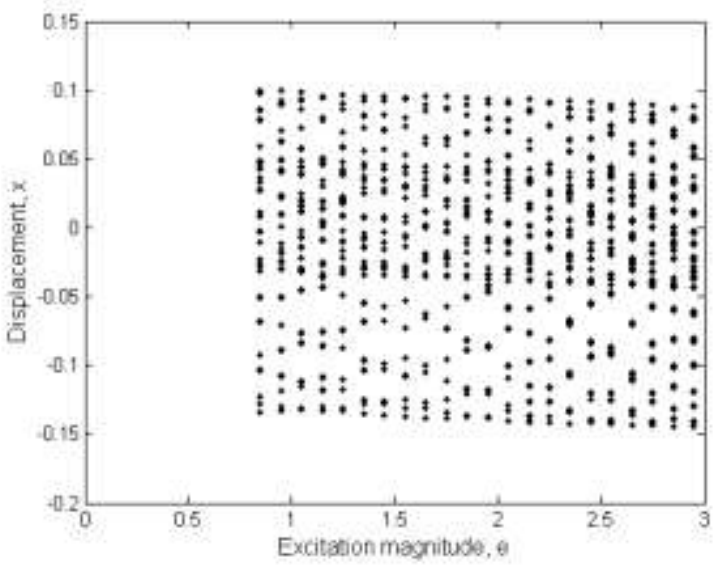

(b) bifurcation diagram at $\Omega=6.094$

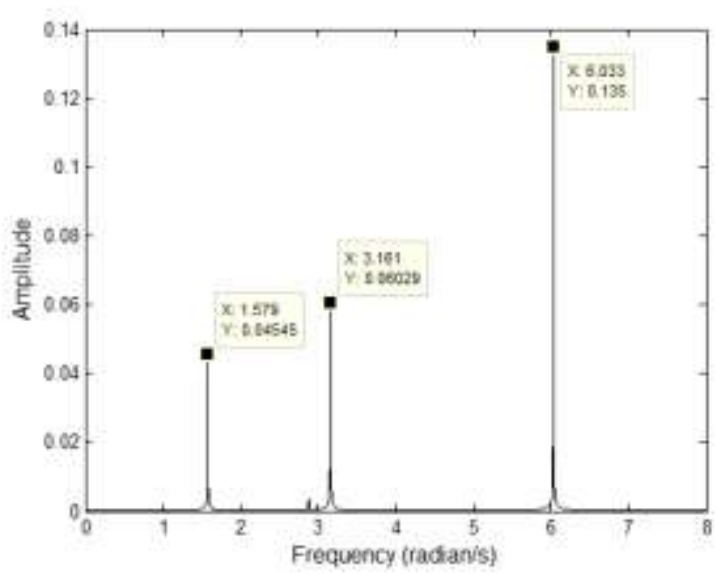



(c) time history at $\Omega=6.034, e=3.5$
(d) frequency spectrum at $\Omega=6.034, e=3.5$

Fig. 7 The forced response at two times the higher SHBF; (a) bifurcation diagram at $\Omega=6.034$, (b) bifurcation diagram at $\Omega=6.094$, (c) time history, and (d) frequency spectrum at $\Omega=6.034, e=3.5$.

Interestingly, the forced response demonstrates the small QP motion, which is qualitatively different from the corresponding segment shown in Figure 4(b). As shown in Figure 7(c), this small-amplitude QP motion can also be regarded as non-resonant response and contains three dominant frequency components, which do not hold the relationship of sub-harmonic resonances. Surprisingly, the SHBF components previously existing in the large-amplitude non-resonant quasi-periodic motion disappear and the $H B F s$ reappear in the resultant non-resonant response after the forcing frequency is adjusted at two times the higher SHBF. It is conjectured that the small-amplitude $S B S$ is excited and the response is actually a direct interaction between the particular solution and the small-amplitude SBS. As discussed in reference [23], the two SBSs of the corresponding autonomous time-delayed nonlinear system have their own basin of attraction. Under the periodic excitation, the basin of attraction may be changed depending on the forcing frequency and magnitude, thus leading to different motion. In summary, under same initial conditions, the large QP motion under two times the higher $H B F$ will disappear and becomes the small QP motion if the forcing frequency is changed according to the frequency components of the large-amplitude SBS. This indicates that the sub-harmonic resonance at two times the higher SHBF cannot be established halfway through by tuning the forcing frequency.

\section{The family of sub-harmonic resonance at two times the higher SHBF}

The forcing frequency is initially set in the neighbourhood of two times the higher SHBF. Figure 8 shows the bifurcation diagrams of the sub-harmonic resonance response for four different excitation frequencies. For $\Omega=5.974$, as shown in Figure 8(a), the forced response demonstrates large QP motion over the entire region of the forcing amplitude. For $\Omega=5.994$, the bifurcation diagram shown in Figure $8(b)$ is qualitatively different from the one at $\Omega=5.974$, and demonstrates large QP motion for $e<2.15$ and periodic motion for $e>2.15$. When the forcing frequency is changed to two times the higher $S H B F$, the forced response, as given in Figure 8(c), admits large-amplitude periodic motion whose period is four times the period of the periodic excitation. The bifurcation diagram shown in Figure $8(\mathrm{~d})$ for $\Omega=6.094$ can be divided into three regions, namely, $0.05 \leq e \leq 1.75$, 
$1.75<e \leq 2.65$, and $2.65<e \leq 3.0$. Correspondingly, the time-delayed system exhibits large QP motion, a window of large-amplitude periodic and QP motion, and largeamplitude periodic motion, respectively.

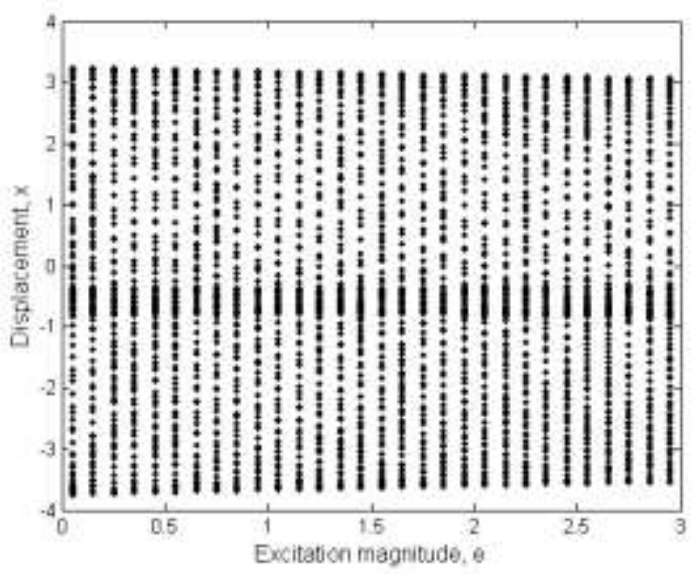

(a) $\Omega=5.974$

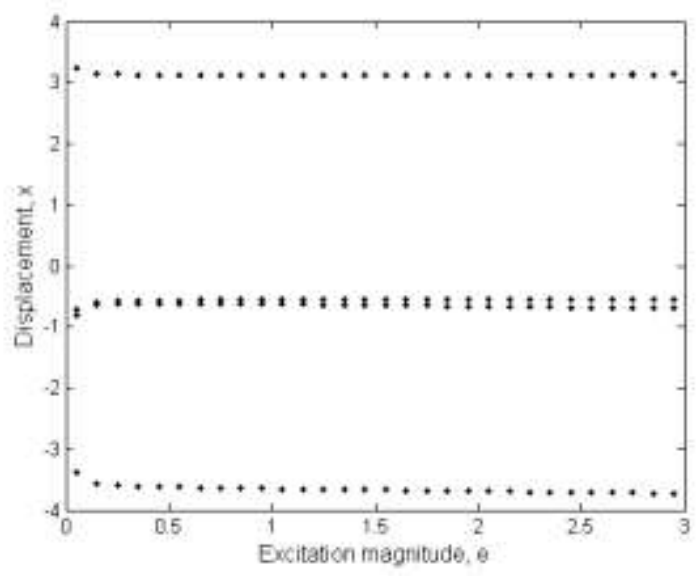

(c) $\Omega=6.034$

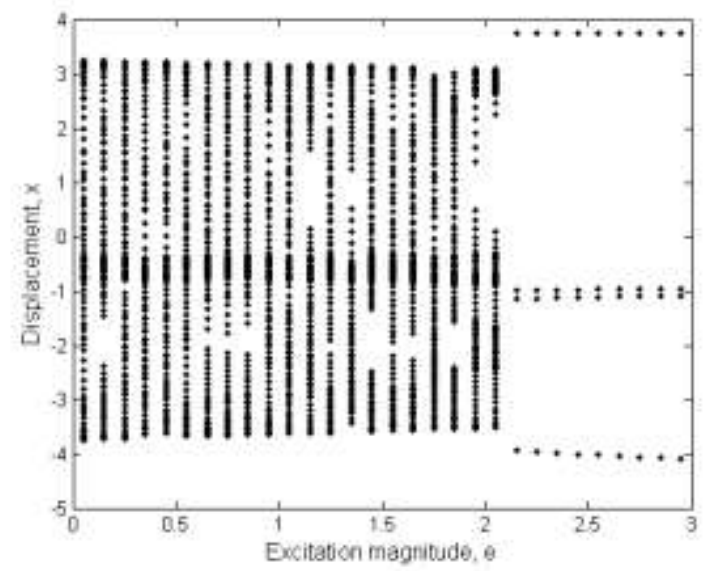

(b) $\Omega=5.994$

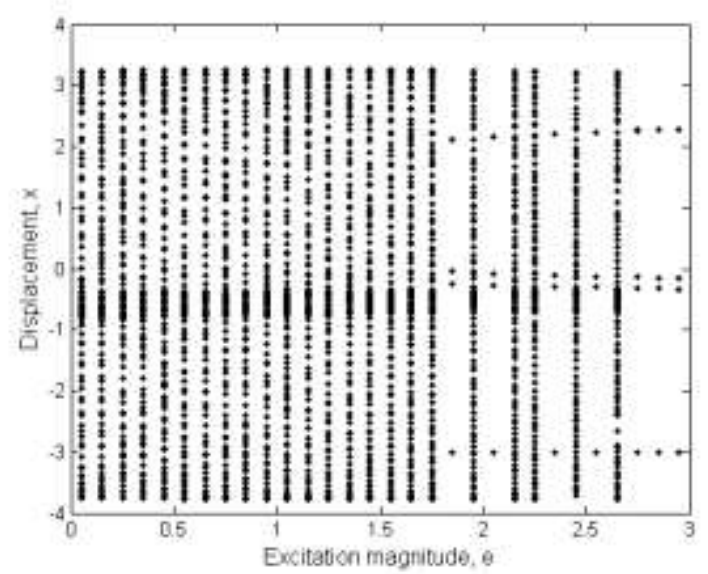

(d) $\Omega=6.094$

Fig.8. Bifurcation diagrams of the sub-harmonic resonance response at two times the higher SHBF. (a) $\Omega=5.974$, (b) $\Omega=0.7485$, (c) $\Omega=6.034$, (d) $\Omega=6.094$.

Figure 9 shows time trajectories and frequency spectra of the sub-harmonic resonance response at two times the higher $S H B F$, where the frequency and magnitude of the periodic excitation are chosen in line with Figure 8(d). Two values of the excitation amplitudes are taken in the window region of large-amplitude periodic and QP motion. At $e=2.25$, the large QP motion has four main frequency components which are two $S H B F s$, the sum of two SHBFs, and the forcing frequency. There are also extra peaks around the higher SHBF and the forcing frequency, as shown in Figure 9(a). A closed curve is shown on the Poincare map further confirming the forced response is quasi- 
periodic. Figure 9(b) shows the large-amplitude periodic motion for $e=3.5$ having four frequency components, which can be considered as one to four quarters the forcing frequency. It is very interesting to note that the amplitude of the large-amplitude periodic motion does not change significantly as the forcing magnitude increases. However, the magnitudes of the frequency components have changed, indicating the occurrence of sub-harmonic resonance at two times the higher SHBF.
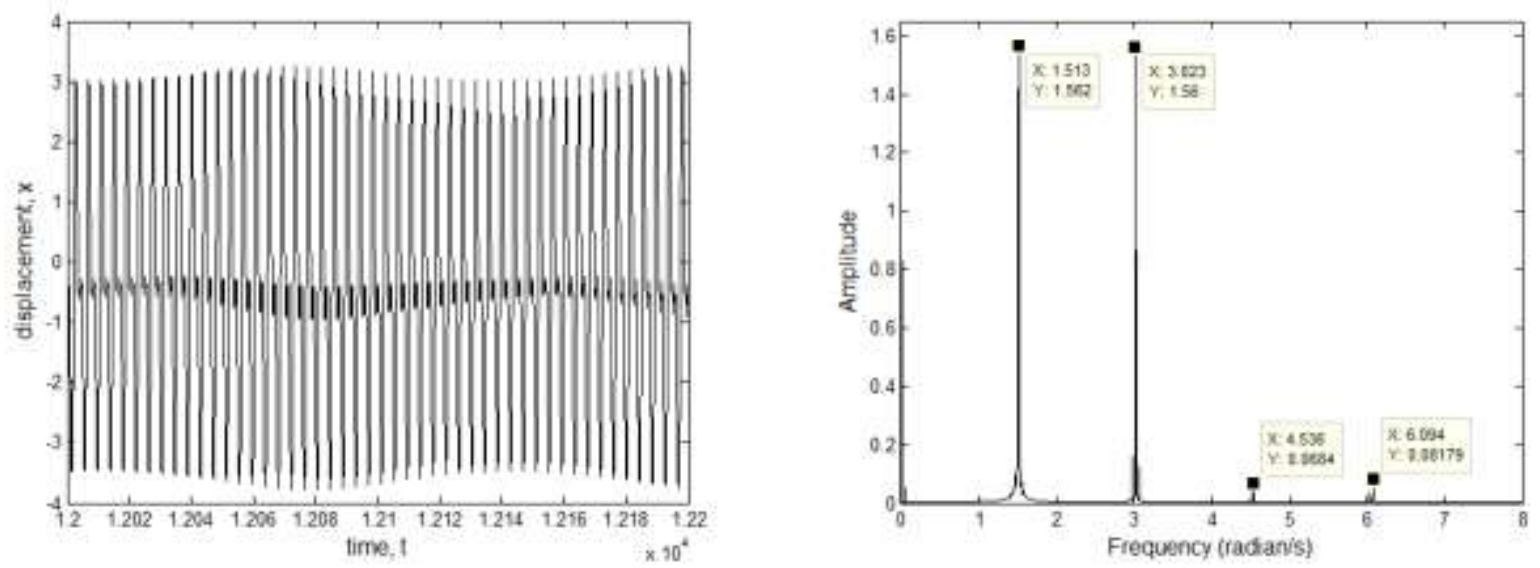

(a) $e=2.25$
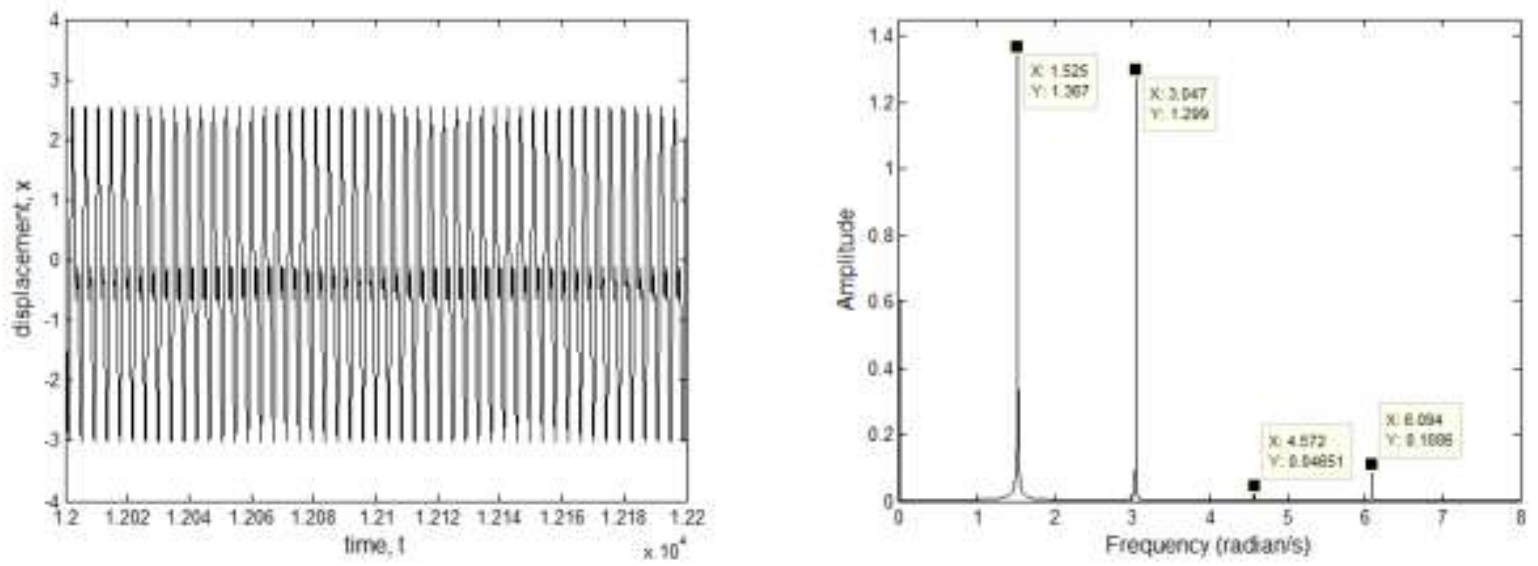

(b) $e=3.5$

Fig.9. Time trajectories and frequency spectra of the forced response under the subharmonic resonance related to the SHBFs, (a) $e=2.25$, (b) $e=3.5$.

\section{Conclusion}

This paper identified two coexisting families of sub-harmonic resonances at different frequencies in the forced response of a time-delayed nonlinear system, when two coexisting stable bifurcating solutions are present in the corresponding autonomous systems. Under the family of sub-harmonic resonance at two times the higher the $H B F$, 
the forced response may demonstrate small- and large-amplitude QP motion. On the contrary, under the family of sub-harmonic resonance at two times the higher the SHBF, the forced response may show large-amplitude QP and periodic motion, as well as the window of large-amplitude periodic motion between QP motion. The phenomenon of coexisting sub-harmonic resonances is a distinctive feature for the time-delayed singledegree-of-freedom nonlinear system and does not have an analogy in the corresponding classical single-degree-of-freedom nonlinear system with only one periodic excitation.

The initially established sub-harmonic resonance related to the HBFs can be lost with the disappearance of the HBF components, and large-amplitude QP motion containing the SHBFs appears in the non-resonant response. On the contrast, the initially established sub-harmonic resonance resonances associated with the SHBFs does not vanish in the forced response. Following the loss of the sub-harmonic resonances associated with the HBFs, the sub-harmonic resonances related to the SHBFs cannot be established halfway through even by purposely changing the forcing frequency to be about two times the higher SHBF. After doing so, the large-amplitude non-resonant motion becomes the small-amplitude quasi-periodic motion.

\section{References}

[1] J.C. Ji, C.H. Hansen, Hopf bifurcation of a magnetic bearing system with time delay, Journal of Vibration and Acoustics-Transactions of the ASME, vol.127(4) (2012), pp.362-369.

[2] T. Kalmar-Nagy, G. Stepan, F.C. Moon, Subscritical Hopf bifurcation in the delay equation model for machine tool vibrations, Nonlinear Dynamics, vol.26 (2001), pp.121142.

[3] D.E. Gilsinn, Estimating critical Hopf bifurcation parameters for a second-order delay differential equation with application to machine tool chatter, Nonlinear Dynamics, vol.26 (2002), pp.103-154.

[4] P. Wahi, A. Chatterjee, Regenerative tool chatter near a co-dimension 2 Hopf point using multiple scales, Nonlinear Dynamics, vol.40 (2005), pp.323-338.

[5] Z. Liu, G. Payre, Stability analysis of doubly regenerative cylindrical grinding process, Journal of Sound and Vibration, vol.301 (2007), pp.950-962. 
[6] K.J. Kalinski, M.A. Galewski, Chatter vibration surveillance by the optimal-linear spindle speed control, Mechanical Systems and Signal Processing, vol.25(1)(2011), pp.383-399.

[7] S. Shao, K.M. Masri, M.I. Younis, The effect of time-delayed feedback controller on an electrically actuated resonator, Nonlinear Dynamics, vol.74 (1-2) (2013), pp.257-270.

[8] P. Ramachandran, Y.M. Ram, Stability boundaries of mechanical controlled system with time delay, Mechanical Systems and Signal Processing, vol.27(2012), pp.523-533.

[9] G.I.K. Taffo, M.S. Siewe, Parametric resonance, stability and heteroclinic bifurcation in a nonlinear oscillator with time-delay: application to a quarter-car model, Mechanics Research Communications, vol.52 (2013), pp.1-10.

[10] Z.H. Liu, K. Hu, K.W. Chung, Nonlinear analysis of a closed-loop tractor-semitrailer vehicle system with time delay, Mechanical Systems and Signal Processing, vol.7677(2016), pp.696-711.

[11] M. Nazari, E.A. Butcher, Analysis of stability and Hopf bifurcation of delayed feedback spin stabilization of rigid spacecraft, Nonlinear Dynamics, vol.74(3) (2013), pp.801-817.

[12] B. Balachandran, T. Kalmar-Nagy, D.E. Gilsinn, Delay Differential Equations Recent Advances and New Directions. Springer, New York (2009)

[13] J.C. Ji, Nonresonant Hopf bifurcations of a controlled van der Pol-Duffing oscillator, Journal of Sound and Vibration, vol.297 (2006), pp.183-199.

[14] J. Xu, P. Yu, Delay-induced bifurcations in a nonautonomous system with delayed velocity feedbacks, International Journal of Bifurcation and Chaos, vol.14(8) (2004), pp.2777-2798.

[15] Z. Dadi, Z. Afsharnezhad, N. Pariz, Stability and bifurcation analysis in the delaycoupled nonlinear oscillators, Nonlinear Dynamics, vol.70(1) (2012), pp.155-169.

[16] R.D. Naik, P.M. Singru, Stability and Hopf bifurcation of a nonlinear oscillator with multiple time-delays, Chaos Solitons \& Fractals, vol.45(11) (2012), pp.1387-1396.

[17] H. Zang, T.H. Zhang, Y.D. Zhang, Stability and bifurcation analysis of delayed coupled van der Pol-Duffing oscillators, Nonlinear Dynamics, vol.75(1-2) (2014), pp.3547. 
[18] K.A. Alhazza, M.F. Daqaq, A.H. Nayfeh, D.J. Inman, Non-linear vibrations of parametrically excited cantilever beams subjected to non-linear delayed-feedback control, International Journal of Non-Linear Mechanics, vol.43(8) (2008), pp.801-812.

[19] M.F. Daqaq, K.A. Alhazza, Y. Qaroush, On primary resonances of weakly nonlinear delay systems with cubic nonlinearities, Nonlinear Dynamics, vol.64 (2011), pp.253277.

[20] H. Moradi, M.R. Movahhedy, G. Vossoughi, Bifurcation analysis of milling process with tool wear and process damping: regenerative chatter with primary resonance, Nonlinear Dynamics, vol.70 (2012), pp.481-509.

[21] M.S. Siewe, C. Tchawoua, S. Rajasekar, Parametric resonance in the RayleighDuffing oscillator with time-delayed feedback, Communications in Nonlinear Science and Numerical Simulation, vol.17(1) (2012), pp.4485-4493.

[22] J.C. Ji, N. Zhang, W. Gao, Difference resonances in a controlled van der Pol-Duffing oscillator involving time delay, Chaos, Solitons and Fractals, vol.42 (2009), pp.975-980.

[23] J.C. Ji, Two families of super-harmonic resonances in a time-delayed nonlinear oscillator, Journal of Sound and Vibration, vol.349(2015), pp.299-314.

[24] T. Kapitaniak, Combined bifurcations and transition to chaos in a non-linear oscillator with two external periodic forces, Journal of Sound and Vibration, vol.121(2) (1988), pp.259-268.

[25] J.C. Ji, Secondary resonances of a quadratic nonlinear oscillator following two-to-one resonant Hopf bifurcations, Nonlinear Dynamics, vol.78(3) (2014), pp.2161-2184.

[26] J.C. Ji, et al:: Two-to-one resonant Hopf bifurcations in a quadratically nonlinear oscillator involving time delay, International Journal of Bifurcation and Chaos, vol.22(3) (2012), Article number: 1250060.

[27] J.K. Hale, S.M. Verduyn lunel, Introduction to Functional Differential Equations. Springer-Verlag, New York (1993).

[28] A.H. Nayfeh, D.T. Mook, Nonlinear Oscillations. Wiley, New York (1979) 